Revue

Revue de l'histoire des religions

del'histoire des religions

\title{
Didier KAHN (éd.), La Messe alchimique attribuée à
}

Melchior de Sibiu

Paris, Classiques Garnier (« Textes de la Renaissance », 197), 2015

\section{Bruno Restif}

\section{OpenEdition}

\section{Journals}

Édition électronique

URL : http://journals.openedition.org/rhr/8699

DOI : ERREUR PDO dans /localdata/www-bin/Core/Core/Db/Db.class.php L.34 : SQLSTATE[HYO0O]

[2006] MySQL server has gone away

ISSN : 2105-2573

Éditeur

Armand Colin

Édition imprimée

Date de publication : 1 mars 2017

Pagination : 170-173

ISBN : 978-2-200-93125-4

ISSN : 0035-1423

\section{Référence électronique}

Bruno Restif, «Didier KaHn (éd.), La Messe alchimique attribuée à Melchior de Sibiu », Revue de l'histoire des religions [En ligne], 1 | 2017, mis en ligne le 24 mars 2017, consulté le 07 janvier 2021. URL : http:// journals.openedition.org/rhr/8699 ; DOI : https://doi.org/ERREUR PDO dans /localdata/www-bin/ Core/Core/Db/Db.class.php L.34 : SQLSTATE[HY000] [2006] MySQL server has gone away

Ce document a été généré automatiquement le 7 janvier 2021

Tous droits réservés 


\section{Didier KAHN (éd.), La Messe} alchimique attribuée à Melchior de

\section{Sibiu}

Paris, Classiques Garnier (« Textes de la Renaissance », 197), 2015

\section{Bruno Restif}

\section{RÉFÉRENCE}

Didier KAHN (éd.), La Messe alchimique attribuée à Melchior de Sibiu, Paris, Classiques

Garnier («Textes de la Renaissance », 197), 2015, 22 cm, 149 p., $29 €$, ISBN

978-2-8124-3274-3.

Ce petit livre s'inscrit dans la lignée des travaux consacrés par D. Kahn à l'alchimie depuis la soutenance de sa thèse en 1998, dont l'essentiel du texte a été publié par la Librairie Droz en 2007 sous le titre Alchimie et paracelsisme en France à la fin de la Renaissance (1567-1625). Après plusieurs éditions de textes alchimiques, l'auteur s'attaque enfin à cette "messe alchimique ", " seule de son espèce ", qu'il évoquait dès 1998. Il s'agit avant tout d'une édition critique, établie avec le concours d'Alena Hadravova (Prague) et de Jean-Baptiste Lebigue (IRHT, Paris), à la suite d'éditions tchèques jugées insuffisamment complètes (p. 28). L'établissement du texte s'est fait à partir des versions imprimées (en 1597 et 1608) et manuscrites (datant de la fin du XVI et de la première moitié $\mathrm{du} \mathrm{XVII}^{\mathrm{e}}$ siècle), en tchèque, allemand et latin, qui sont ellesmêmes des copies puisque le texte original daterait du milieu du $\mathrm{xv}^{\mathrm{e}}$ siècle. Son auteur serait un ecclésiastique transylvanien nommé Melchior de Sibiu, qui aurait été proche d'un roi de Bohême et de Hongrie, peut-être Ladislas le Posthume, peut-être aussi du dernier roi de Bosnie Étienne Tomašević (ou bien cet auteur aurait transcrit des formules communiquées par ce dernier), et qui aurait probablement été exécuté à Prague comme faux-monnayeur (du fait de son activité alchimique), puis à qui Carl Gustav Jung s'est intéressé au $\mathrm{Xx}^{\mathrm{e}}$ siècle. 
2 D. Kahn édite les textes latin et tchèque, en indiquant les variantes et en donnant une traduction française. L'analyse liturgique du texte est menée, avec l'aide de J.-B. Lebigue, de façon serrée pour en établir les cohérences et éventuelles incohérences. D. Kahn s'interroge ensuite sur la signification possible des variantes en lien avec la transmission du texte. En effet, l'un des aspects les plus intéressants de l'énigme est cette transmission, assurée à la fois par un calviniste français devenu socinien, un catholique tchèque, un schwenckfeldien allemand et deux pasteurs luthériens allemands dont l'un est devenu paracelsien, ce qui montre la «capacité de l'alchimie à se jouer des frontières religieuses». D. Kahn éprouve d'ailleurs les plus grandes difficultés à identifier les modifications que les uns et les autres auraient pu apporter à cette messe pour des raisons théologiques, mais il faut considérer d'une part que plusieurs d'entre eux sont des hétérodoxes, d'autre part que leur volonté de copier ce texte leur impose d'en respecter l'essentiel. Au XvII siècle, le texte de cette messe, désormais imprimé, est loué, non sans quelque réserve, par l'alchimiste Michael Maier et pour cette raison il intègre la tradition rosicrucienne, tandis qu'il est condamné par le luthérien Libavius et par les jésuites Garasse et Kircher.

3 La critique déployée dans cet ouvrage souligne l'impossibilité de rattacher cette messe «à une liturgie particulière » ou de façon certaine à un moment précis du calendrier liturgique, même si des hymnes et antiennes désignent plutôt l'Avent et Noël. Mais pourrait-il en être autrement et à quel autre moment du calendrier pourrait-il être logique d'insérer une messe alchimique? Plus intéressantes sont les remarques de J.-B. Lebigue sur la difficulté à articuler les formules rythmiques des textes mentionnés et des airs sur lesquels ils doivent être chantés. Ceci dit, cette incohérence apparente n'est pas aisée à expliquer car d'autres éléments prouvent la maîtrise liturgique de l'auteur du texte, peut-être chapelain de Ladislas le Posthume. En outre, ces difficultés rythmiques n'ont pas empêché la célébration de cette messe en 1943 et son adaptation récente par un ensemble de musique contemporaine (p. 27). La consécration n'apparaît pas, et c'est évidemment le contraire qui aurait été fort surprenant.

Le texte lui-même permet une double lecture, chrétienne et alchimique, à propos de l'eau, de la terre, du soleil ou feu, de la guérison, de la fécondation d'une vierge, tandis qu'est développé un parallèle entre le Christ et la pierre philosophale. Aussi l'hypothétique célébration de cette messe pendant l'Avent présenterait-elle une certaine logique. L'éditeur du texte relève la nette influence du Flos florum faussement attribué à Arnaud de Villeneuve dans le choix des textes. Forme d'hapax, cette messe alchimique présente donc, tout autant que sa transmission, un grand intérêt pour l'historien, et elle pose sans doute plus de questions qu'elle n'offre de réponses, malgré le grand soin apporté à la présente édition.

5 De fait, il semble possible de discuter les interprétations proposées par D. Kahn, surtout lorsqu'il écrit que cette messe "prend [...] son sens lorsqu'on la replace [...] dans le contexte médiéval des messes parodiques et des messes votives" (quatrième de couverture, affirmation développée p. 39). La référence à la parodie est surprenante, car on imagine mal les alchimistes se livrer à une fête des fous en parodiant leur savoir et leurs techniques : le texte de la messe alchimique nous apparait au contraire comme tout à fait sérieux. Le renvoi aux messes votives semble en contradiction avec l'explication parodique, il peut convaincre toutefois à condition d'accorder une validité au rituel et de ne pas faire de cette messe un pur « objet littéraire » et même un «jeu littéraire » (p. 126), ce que fait précisément D. Kahn. La logique de l'argumentation peut 
donc dérouter, voire laisser sceptiques certains lecteurs. Le refus de faire de cette «messe " un rituel est justifié par des arguments liturgiques, précisés plus haut mais qui - comme nous l'avons exposé - ne nous convainquent pas, par la forme littéraire et poétique de la composition (ce qui peut être considéré comme n'étant pas un argument puisque la production liturgique présente les mêmes caractéristiques), et peut-être surtout par la volonté de dégager l'alchimie de toute accusation d'hérésie (p.9) et de magie. Ainsi, selon l'auteur, «les domaines de la magie et de l'alchimie restèrent parfaitement étanches l'un à l'autre tout au long du Moyen Âge, à de très rares exceptions près » (p.39), affirmation qu'une note de bas de page justifie de la façon suivante: "sur les traditions entièrement distinctes de la magie et de l'alchimie, il suffit de se reporter à toute la littérature secondaire récente sur chacun de ces domaines (en excluant bien sûr les études dues à des non-spécialistes)» (p. 39).

Il se pourrait donc que l'interprétation de la messe alchimique comme simple jeu littéraire trouve là son explication essentielle. Dans son ouvrage Alchimie et paracelsisme en France, l'auteur définissait la magie par son irrationalité tandis que l'alchimie devait être considérée comme scientifique parce que la transmutation des métaux était alors «théoriquement possible» (p. 9). Pourtant, ces deux pôles de la «science» et de la «magie " sont historiquement constitués, et donc mouvants, dépendant notamment des discours de légitimation ou de condamnation qu'elles produisent ou qui sont produits sur elles. Dans sa volonté justifiée de rejeter la vision romantique de l'alchimie élaborée au XIX siècle, D. Kahn se laisse ainsi paradoxalement et involontairement influencer par cette forme de scientisme qui a pour partie déterminé l'analyse de la magie à la fin du xIx et dans la première moitié $d u x^{e}$ siècle, comme fausse science ou pensée prélogique. Mais en fonction de quels critères? Ceux de Frazer, Malinowski ou Lévy-Bruhl? Et si la réussite d'un acte dit «magique" n'était pas elle aussi théoriquement possible, quel en serait l'intérêt? En outre, il va de soi pour un ecclésiastique (ce qu'était Melchior de Sibiu) que l'efficacité de la messe est au minimum "théoriquement possible ", ce qui ne saurait donc invalider l'hypothèse du rituel. Pour autant, précisons-le bien, rien n'indique que cette messe alchimique ait été

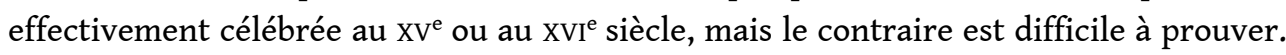
Lorsqu'elle est diffusée à partir de la fin du XvI ${ }^{\mathrm{e}}$ siècle, dans un contexte différent, et pas seulement par des catholiques et des luthériens (qui doivent eux-mêmes composer avec leur orthodoxie confessionnelle), elle semble bien être un " objet littéraire ", mais s'agit-il pour autant d'un «jeu »? On ne peut exclure que le statut accordé au texte ait varié.

7 Dans l'introduction du volume, D. Kahn rappelle l'importance des interprétations alchimiques du texte biblique et de la conception spirituelle qui insiste sur la «transmutation » intérieure de l'opérateur, conception qui «n'existe pas au Moyen Âge » et "n'apparaît qu'à la Renaissance » (p.13). Il y a peut-être là une autre interprétation possible de cette messe alchimique, composée à la charnière du Moyen Âge et de la Renaissance. Et si Melchior de Sibiu était déjà un homme de la Renaissance, une Renaissance que les hétérodoxes ont tenté de faire survivre lorsqu'elle disparaissait? 


\section{AUTEURS}

BRUNO RESTIF

Université de Reims Champagne-Ardenne. 